Pacific Journal of Mathematics

AFFINE LAMINATION SPACES FOR SURFACES

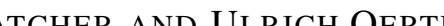




\section{AFFINE LAMINATION SPACES FOR SURFACES}

\section{A. Hatcher and U. Oertel}

In this paper we extend Thurston's space $\mathscr{P} \mathscr{L}(M)$ of projective classes of measured laminations in the compact surface $M$ to a space $\mathscr{A} \mathscr{L}(M)$ of laminations with transverse affine structures. The main theorem is that $\mathscr{A} \mathscr{L}(M)$ is homeomorphic to the product $\mathscr{P} \mathscr{L}(M) \times$ $H^{1}(M ; \mathbb{R})$.

To avoid confusion, it should be pointed out at the outset that the term "affine lamination" can have two different meanings, depending on whether the transverse affine structure is defined in the ambient surface or just in a neighborhood of the lamination. It is the former, stronger, notion that we are interested in here. Such ambient affine structures can be regarded as transverse affine structures on singular foliations of $M$.

The topology on $\mathscr{A} \mathscr{L}(M)$ is defined via length functions on homologically trivial loops in $M$, by the following procedure. The obstruction to an affine lamination $L \in \mathscr{A} \mathscr{L}(M)$ being a measured lamination is a holonomy homomorphism $\sigma_{L}: \pi_{1}(M) \rightarrow \mathbb{R}_{+}$, which measures the amount by which arcs transverse to $L$ are stretched or shrunk as they are transported around loops in $M$. Since $\mathbb{R}_{+}$is abelian, commutators in $\pi_{1}(M)$ have trivial holonomy, so the lift $\widehat{L}$ of $L$ to the universal abelian cover $\widehat{M}$ of $M$, corresponding to the commutator subgroup of $\pi_{1}(M)$, has a transverse measure, unique up to scalar multiplication. Let $\widehat{\mathscr{A} \mathscr{L}}(M)$ denote the unprojectivized version of $\mathscr{A} \mathscr{L}(M)$, consisting of the measured laminations $\widehat{L} \subset \widehat{M}$ constructed in this way. Loops $\gamma$ in $\widehat{M}$ determine length functions $l_{\gamma}: \widehat{\mathscr{A} \mathscr{L}}(M) \rightarrow[0, \infty)$, with $l_{\gamma}(L)$ as usual the infimum of the length, with respect to the transverse measure on $\widehat{L}$, of loops in $\widehat{M}$ homotopic to $\gamma$. These $l_{\gamma}$ 's are the coordinates of a function $l: \widehat{\mathscr{A L}}(M) \rightarrow[0, \infty)^{\mathscr{C}}, \mathscr{C}$ being the set of free homotopy classes of loops in $\widehat{M}$. We prove $l$ is injective, and give $\widehat{\mathscr{A} \mathscr{L}}(M)$ the induced topology. Projectivizing this yields $\mathscr{A} \mathscr{L}(M)$.

We then determine the global topology of $\mathscr{A} \mathscr{L}(M)$. The most 
natural statement is that the holonomy map

$$
\sigma: \mathscr{A} \mathscr{L}(M) \rightarrow \operatorname{Hom}\left(\pi_{1}(M), \mathbb{R}_{+}\right) \approx H^{1}(M ; \mathbb{R}), \quad L \mapsto \sigma_{L},
$$

is a fiber bundle. The fiber over the trivial holonomy is $\mathscr{P} \mathscr{L}(M)$, and the base space is contractible, so it follows that $\mathscr{A} \mathscr{L}(M)$ is homeomorphic to the product $\mathscr{P} \mathscr{L}(M) \times H^{1}(M ; \mathbb{R})$. However, there does not appear to be a natural projection of $\mathscr{A} \mathscr{L}(M)$ onto the fiber $\mathscr{P} \mathscr{L}(M)$, and so the product structure may not be a natural one.

An interesting problem which we barely touch upon is the structure of individual affine laminations, in particular how they differ from measured laminations.

1. Preliminaries on laminations. In this section we generalize three well-known facts about measured laminations in surfaces to nonmeasured laminations.

Consider first foliations of a compact, connected surface $M$ of negative Euler characteristic, with leaves meeting $\partial M$ transversely, and with isolated $n$-prong singular points in $M-\partial M, n \geq 3$. Such a foliation $F$ can be de-singularized to a lamination $L$ by splitting open along the leaves which meet the singular points. The restriction on the singularity types is equivalent to the condition that the regions of $M-L$ have negative index, where "index" is measured by a line field on $M$ tangent to $L$, transverse to $\partial M$, and with isolated singularities in $M-L$. Unless otherwise stated, all laminations in this paper will be understood to be constructible in this way; in particular, they will have no isolated leaves.

If we split a foliation $F$ only along compact subarcs of all the singular leaves, subarcs with one or both endpoints at singular points, we obtain an intermediate notion which we call a prelamination. This is a foliation of a compact subsurface of $M$ whose singularities are cusps (as in a train track). A lamination can be regarded as an equivalence class of prelaminations under the equivalence relation generated by further compact splitting. Note that if we form a prelamination by splitting along arcs which contain all the compact singular leaves, we obtain a canonical representative in each equivalence class, unique up to isotopy, since further compact splitting is then realizable by isotopy.

By a Reeb component in a foliation, lamination, or prelamination in $M$ we mean either the usual foliated annulus or, when $M$ is nonorientable, the analogous foliated Möbius band. A $\partial$-Reeb component is a foliated annulus with one boundary circle a component of $\partial M$ transverse to the foliation, the leaves meeting this circle being rays spi- 
ralling towards the other boundary circle, which is a leaf in $M-\partial M$. A $\partial$-Reeb component is thus "half of a Reeb component." Splitting a foliation open to a prelamination or a lamination, in the way described above, has no effect on Reeb or $\partial$-Reeb components since these are foliated without singularities. Note also that $\partial$-Reeb components exist iff $\partial$-parallel leaves exist, since a $\partial$-parallel leaf cuts off an annulus which must be foliated non-singularly, and must therefore meet $\partial M$ in a $\partial$-Reeb component.

We call a train track in $M$ good if all its complementary regions have negative index.

Proposition 1.1. A lamination in $M$ has no Reeb or $\partial$-Reeb components iff it is representable by a prelamination which is a foliated neighborhood of a good train track, with leaves transverse to the interval fibers of a fibered neighborhood of the train track.

Proof. Suppose first that the prelamination $L$ is such a foliated neighborhood $N(\tau)$ of a good train track $\tau$, and that $L$ contains a Reeb or $\partial$-Reeb component $R$. Consider an interval fiber of $N(\tau)-$ $\partial M$ crossing $\partial R-\partial M$. This interval enters $R$ and stays transverse to the leaves of $R$, so it can never come out to $\partial R-\partial M$ again, which contradicts the fact that it is a compact interval with endpoints on $\partial L-\partial M$.

Conversely, suppose $L$ is a prelamination without Reeb or $\partial$-Reeb components. Let $\nu$ be a line field on $L$ transverse to leaves. If every trajectory of $\nu$ is cut into compact segments by the singular leaves of $L$, then we are done, since by compactness a finite splitting of $L$ cuts all trajectories of $\nu$ into segments, so collapsing these gives the train track. If some trajectory of $\nu$ is not cut into compact segments, then one end of this trajectory must be disjoint from singular leaves. From an accumulation point of this end one can produce a circle $C$ in $L$ transverse to leaves and disjoint from all singular leaves. Now split $L$ open to get an actual lamination $L^{\prime}$. The path-component $A$ of $L^{\prime}$ containing $C$ is a foliated surface. If $A$ is noncompact, its ends are "cusps" $I \times[0, \infty)$ with the product foliation; this is clear from the process of splitting $L$ open to form $L^{\prime}$. Since $L$ has only finitely many singular leaves, $A$ has finitely many cusps. Thus the usual line-field index is defined for $A$, and must be zero since $A$ is foliated. This forces $A$ to be $I \times(-\infty, \infty), I \times[0, \infty), I \times I$, $I \times S^{1}$, or a Möbius band. Since $A$ contains the circle $C$ transverse to leaves, it must be nonsimply-connected, hence an annulus or Möbius 
band. Then if $A$ contains no Reeb or $\partial$-Reeb components, it must be foliated as a suspension (or mapping torus) of a homeomorphism $I \rightarrow I$. By redefining $\nu$ in $A$ we can eliminate all transverse circles $C$ in $A$. Since $L^{\prime}$ can contain only finitely many such foliated surfaces $A$ (each $A$ contains one of the finitely many boundary leaves of $L^{\prime}$ ), this process is eventually completed.

Let $C$ be a union of finitely many disjoint circles in $M$. We say $C$ meets a lamination or prelamination $L$ minimally if $C$ is transverse to $L$ and there is no disk $D \subset M$ with $\partial D$ consisting of an arc in $C$ and an arc in a leaf of $L$; passing to an innermost such disk, we may assume $\operatorname{int}(D)$ is disjoint from $C$ and $L$.

Proposition 1.2. If $L$ is a lamination whose Reeb components are all d-parallel, then $C$ can be isotoped to meet $L$ minimally.

If $L$ has non- $\partial$-parallel Reeb components, minimal position is clearly impossible for certain curves $C$.

In terms of prelaminations, the proposition is equivalent to saying that a prelamination with Reeb components all $\partial$-parallel can be split (along singular leaves, as always) and isotoped to meet $C$ minimally.

Proof. First we can immediately reduce to the case of no Reeb or $\partial$-Reeb components by deleting from $M$ neighborhoods of the annuli cut off by $\partial$-parallel leaves of $L$. Then by the previous proposition, we can embed the lamination $L$ in a fibered neighborhood $N$ of a good track, transverse to fibers.

Consider circle systems $C$, isotopic to the given one, which are divided into finitely many segments lying either outside $N$, in fibers of $N$ ("vertical"), or in $N-L$ transverse to fibers ("horizontal"). Such a segmented $C$ can be produced for example by making $C$ transverse to the train track obtained by collapsing fibers of $N$ to points, then expanding these points back to fibers, turning the intersections of $C$ with the track into vertical segments. (This $C$ has no horizontal segments.) Define the complexity of a segmented $C$ to be the pair (\#segments outside $N$, \#segments inside $N$ ). Order complexities lexicographically and choose a segmented $C$ of minimum complexity within its isotopy class. Now pull $C$ taut by taking each horizontal segment $\sigma$ having vertical segments at its ends both lying on the same side of $\sigma$, say below $\sigma$, and pushing $\sigma$ downward across leaves of $L$, shortening the two adjacent vertical segments, until some fiber of $N$ meeting $\sigma$ meets no more leaves of $L$ below $\sigma$. This can 
be done keeping $C$ embedded provided we partially order horizontal segments according to the sets of fibers of $N$ which they meet, and pull "smallest" horizontal segments taut first. Pulling $C$ taut is clearly a finite process since each horizontal segment needs to be pulled taut only once.

The claim is that a taut $C$ meets $L$ minimally. For consider a disk $D$ meeting $C$ in an $\operatorname{arc} \alpha \subset \partial D$ and $L$ in an $\operatorname{arc} \beta \subset \partial D$ in a leaf, with $\alpha \cup \beta=\partial D$. Since $C$ has minimum complexity, $\alpha$ must lie in $N$ and contain a single horizontal segment joining the two vertical segments at its ends; $\alpha$ cannot be a single vertical segment by index considerations. Also by index considerations, the horizontal segment in $\alpha$ must traverse the same fibers of $N$ as $\beta$. But then this segment is not taut.

Proposition 1.3. If $P$ is a pair of pants and $L \subset P$ is a lamination without Reeb or $\partial$-Reeb components, then all leaves of $L$ are compact intervals.

Proof. Circles in $P$ either bound disks or are $\partial$-parallel, so $L$ can have no circle leaves. If $L$ has a noncompact leaf $\lambda$, consider the set of limit points of $\lambda$. This forms a sublamination $L^{\prime} \neq \varnothing$. (This $L^{\prime}$ may not be a "lamination" in the restricted sense of this paper since it may not be constructible by splitting open a singular foliation.) $L^{\prime}$ must be disjoint from $\partial P$, since $\lambda$ can meet $\partial P$ in at most one point. $L^{\prime}$ is then carried by a train track disjoint from $\partial P$, a subtrack of a good track carrying $L$ and therefore also good. But $P$ can contain no good track disjoint from $\partial P$, by index considerations: Each of the three boundary circles of $P$ contributes an integer $\leq-1$ to the linefield index of $P$, which is -2 .

2. Affine laminations. A transverse affine structure on a foliation $F$ (with singularities, as above) assigns to each path in $M$ transverse to leaves of $F$ an affine structure which is invariant under homotopies of the path staying transverse to leaves and with endpoints not crossing leaves. Further, the affine structure on subpaths should be obtained by restriction. (An affine structure on a path is an equivalence class of parametrizations related to each other by affine maps of the domain intervals in $\mathbb{R}$.)

Let $\widetilde{F}$ be the lift of the foliation $F \subset M$ to the universal cover $\widetilde{M}$. A transverse affine structure on $F$ lifts to a transverse affine structure on $\widetilde{F}$. Since $\widetilde{M}$ is simply-connected, the transverse affine 
structure on $\widetilde{F}$ underlies a transverse Euclidean structure, unique up to scalar multiplication, obtained by choosing a length for one transverse segment, then extending by "analytic continuation" to lengths for all transverse segments. Each deck transformation of $\widetilde{M}$ takes the Euclidean structure on $\widetilde{F}$ to a scalar multiple of itself, a scalar multiple not depending on the choice of Euclidean structure. Conversely, a projective class of Euclidean structures on $\widetilde{F}$ for which deck transformations act as scalar multiplication determines an affine structure on $F$. This gives an alternative way of defining affine structures in terms of the more familiar Euclidean structures, i.e., transverse measures.

We apply this alternative definition of affine structure to laminations and prelaminations as well as foliations. Thus an affine structure on a lamination $L \subset M$ is a projective class of transverse measures on the lift $\widetilde{L} \subset \widetilde{M}$ such that deck transformations act as scalar multiplication of the measure.

Associating to each $\gamma \in \pi_{1}(M)$ the scalar multiplication factor for the associated deck transformation gives the stretch homomorphism, or holonomy $\sigma_{L}: \pi_{1}(M) \rightarrow \mathbb{R}_{+}$for an affine lamination $L$. Since the multiplicative group of positive reals $\mathbb{R}_{+}$is abelian, $\sigma$ factors through $H_{1}(M)$ and so can be thought of as an element of $H^{1}\left(M ; \mathbb{R}_{+}\right)=$ $\operatorname{Hom}\left(H_{1}(M), \mathbb{R}_{+}\right)$. Since the holonomy homomorphism $\sigma_{L}$ vanishes on the commutator subgroup of $\pi_{1}(M)$, the lift $\widehat{L}$ of $L$ to the abelian cover $\widehat{M}$ (corresponding to the commutator subgroup of $\pi_{1}(M)$ ) has a transverse Euclidean structure unique up to scalar multiplication.

Definition. $\mathscr{A} \mathscr{L}(M)$ is the set of isotopy classes of non-empty laminations in $M$ with transverse affine structures, and without Reeb or $\partial$-Reeb components.

A lamination consisting of just a Reeb or $\partial$-Reeb component can be given an affine structure, provided the core circle of the annulus is non-zero in $H_{1}(M)$, so that the holonomy $\sigma$ can be non-trivial on this circle. More generally one could have an affine structure on any number of parallel Reeb component annuli glued edge-to-edge, all spiralling in the same direction. This trivial sort of complication seems uninteresting, so we avoid it by hypothesis.

The structure of a lamination $L \in \mathscr{A} \mathscr{L}(M)$ near a circle leaf $\lambda$ is easily described. Since $\lambda$ is not isolated, the holonomy around $\lambda$ is an affine map of a nontrivial interval, with the point in $\lambda$ possibly an endpoint. If the holonomy is the identity or a reflection, $L$ near $\lambda$ consists of parallel copies of $\lambda$, and otherwise the leaves of $L$ near $\lambda$ all spiral into $\lambda$ in the same direction, from either one or both sides. 
With such spiralling, the holonomy $\sigma$ must be nontrivial on $\lambda$, so $\lambda$ must be a non-bounding circle in $M$.

Similar holonomy considerations show that if $L \in \mathscr{A L}(M)$ has two parallel circle leaves, then these leaves belong to a component of $L$ which is an annulus foliated by parallel circles. Likewise, in the non-orientable case, if a leaf of $L$ bounds a Möbius band, this leaf belongs to a trivially foliated Möbius band component of $L$.

For a lamination $L \in \mathscr{A} \mathscr{L}(M)$ represented by a prelamination foliating a neighborhood of a good track $\tau$, the associated measured lamination $\widehat{L}$ in the abelian cover $\widehat{M}$ is determined by an assignment of weights to the branches of the lifted track $\hat{\tau} \subset \widehat{M}$, deck transformations acting as scalar multiplication of the total weight vector according to the holonomy $\sigma_{L}$. Conversely, every weight vector for $\hat{\tau}$ satisfying the usual branch equations at the branching points of $\hat{\tau}$, with deck transformations acting by scalar multiplication according to a homomorphism $\sigma: H_{1}(M) \rightarrow \mathbb{R}_{+}$, arises in this way from a lamination $L \in \mathscr{A} \mathscr{L}(M)$. To see this, first choose a fundamental domain $D \subset \widehat{M}$ for the covering space $p: \widehat{M} \rightarrow M$, with $\partial D$ having general position intersection with $\hat{\tau}$. The measure on $\hat{\tau}$ gives a measured prelamination foliating $N(\hat{\tau})$ whose intersection with int $(D)$ projects down by $p$ to a measured prelamination in $p(\operatorname{int}(D))$. The discontinuities which this prelamination may have at $p(\partial D)$ can then be elminated by a simple linear stretching or shrinking in the transverse direction.

Here is a construction of affine laminations which break up into finitely many families of parallel leaves-laminations of finite typewith a given holonomy homomorphism $\sigma: \pi_{1}(M) \rightarrow \mathbb{R}_{+}$. Choose a curve system $C$ in $M$ consisting of disjointly embedded circles and arcs with endpoints on $\partial M$, for which all complementary regions have negative index. Let $C^{\prime} \subset C$ consist of the circles for which $\sigma$ has a value different from 1 . Next, choose a collection $A$ of disjointly embedded arcs in $M-C$ with endpoints on $C^{\prime} \cup \partial M$, each arc having at least one endpoint on $C^{\prime}$ and each circle in $C^{\prime}$ containing at least one endpoint of an arc in $A$. Form a train track $\tau$ from $A \cup C$ by making each arc in $A$ flow tangentially into $C^{\prime}$ in the direction given by the orientation of that circle of $C^{\prime}$ which gives a value of $\sigma$ greater than 1 . Assume the collection $A$ is chosen so that all complementary regions of $\tau$ have negative index; such a choice is always possible if $C^{\prime} \neq \varnothing$. Let $\tau_{\sigma}$ be the lift of $\tau$ to the cover $M_{\sigma}$ of $M$ corresponding to the kernel of $\sigma$. The track $\tau_{\sigma}$ consists of circles and arcs projecting 
to $C-C^{\prime}$, lines projecting to $C^{\prime}$, and arcs projecting to $A$. Choose lifts of the components of $C-C^{\prime}$ and $A$, and assign arbitrary positive weights to these chosen lifts. Requiring that the deck transformations multiply weights according to $\sigma$ specifies weights for the other lifts of the components of $C-C^{\prime}$ and $A$. These weights then determine positive weights on the rest of $\tau_{\sigma}$, the lines projecting to $C^{\prime}$, by summing geometric series. Since deck transformations act by scalar multiplication on these weights on $\tau_{\sigma}$, we obtain an affine lamination $L \in \mathscr{A} \mathscr{L}(M)$ with $\sigma_{L}=\sigma$. The circles and arcs of $C-C^{\prime}$ give rise to bands of parallel circle and arc leaves of $L$, and the arcs of $A$ yield bands of parallel non-compact leaves of $L$ spiralling in on the circles of $C^{\prime}$, which are also leaves of $L$.

It is not hard to see that all affine laminations of finite type can be constructed in this way. The key point is that holonomy around closed leaves is affine.

3. Length functions. The set $\mathscr{A} \mathscr{L}(M)$ can be viewed as the projectivization of a set $\widehat{\mathscr{A L}}(M)$ of measured laminations in the cover $\widehat{M}$ for which deck transformations act as scalar multiplication. $\widehat{(\mathscr{A} \mathscr{L}}(M)$ also includes the empty lamination.) Recall the function $l: \widehat{\mathscr{L} \mathscr{L}}(M) \rightarrow$ $[0, \infty)^{\mathscr{C}}$ defined in the introduction, with coordinates the length functions $l_{\gamma}$ for $\gamma$ a loop in $\widehat{M}$.

THEOREM 3.1. $l$ is injective, hence its projectivization $P(l): \mathscr{A} \mathscr{L}(M)$ $\rightarrow P\left([0, \infty)^{\mathscr{C}}\right)$ is also.

Proof. Consider first the case that $M$ is a pair of pants. The cover $\widehat{M}$ is shown in Figure 3.1 below, with the group of deck transformations $\mathbb{Z} \oplus \mathbb{Z}$ acting simply by translations. The three boundary circles of $M$ lift to the three families of parallel boundary curves of $\widehat{M}$.

In $M$ there are six isotopy classes of non-trivial arcs: $\alpha_{1}, \alpha_{2}, \alpha_{3}$ each joining two boundary circles of $M$, and $\beta_{1}, \beta_{2}, \beta_{3}$ each with both endpoints on one boundary circle, subscripts being chosen so that $\alpha_{1}$ meets $\beta_{1}$ in one point. In $\widehat{M}$ the lifts $\hat{\alpha}_{i}$ join upper and lower strands of $\partial \widehat{M}$ at a crossing, while the lifts $\widehat{\beta}_{i}$ join adjacent parallel lines of $\partial \widehat{M}$ across a "bowtie," as shown in the figure. Lifted laminations $\widehat{L} \in \widehat{\mathscr{A L}}(M)$ consist of various bands of parallel $\hat{\alpha}_{i}$ 's and $\widehat{\beta}_{i}$ 's, with thickness given by the measure on $\widehat{L}$, the whole configuration being such that deck transformations act by scalar multiplication.

Since length functions $l_{\gamma}$ are linear on sums of disjoint curves, it will suffice to find for each lift $\hat{\alpha}_{i}$ or $\widehat{\beta}_{i}$ a linear combination of $l_{\gamma}$ 's 


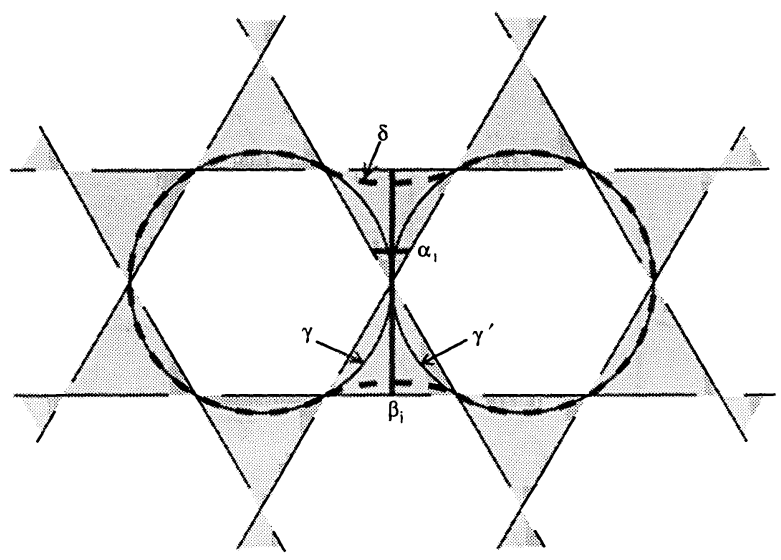

FIGURE 3.1

which is non-zero on that lift and zero on all other lifts of $\alpha_{j}$ 's or $\beta_{j}$ 's. Consider a bowtie containing lifts $\hat{\alpha}_{i}$ and $\widehat{\beta}_{i}$ meeting transversely in one point at the center of the bowtie. This bowtie separates two adjacent hexagons (Figure 3.1) with a common vertex. Let $\gamma$ and $\gamma^{\prime}$ be the loops in $\widehat{M}$ determined by these two hexagons, let $\delta$ be the loop which goes around these two hexagons in succession, both in the same direction (say clockwise), and let $\varepsilon$ be the loop going around the two hexagons in opposite directions, in a figure-eight pattern. Then by inspection one sees that $l_{\gamma}+l_{\gamma}-l_{\varepsilon}$ is non-zero on $\widehat{\beta}_{i}$ but zero on all other lifts $\hat{\alpha}_{j}$ or $\widehat{\beta}_{j}$. Similarly, $l_{\varepsilon}-l_{\delta}$ is non-zero on $\hat{\alpha}_{i}$ and zero on all other lifts $\hat{\alpha}_{j}$, though not on all lifts $\widehat{\beta}_{j}$. This latter defect can be corrected by subtracting appropriate combinations of terms $l_{\gamma}+l_{\gamma}-l_{\varepsilon}$. This finishes the case that $M$ is a pair of pants.

Now we come to the general case. Choose circles $C_{i}$ with neighborhoods $N\left(C_{i}\right)$ decomposing $M$ into pairs of pants $P_{j}$, the components of $M-\cup N\left(C_{i}\right)$. If any $C_{i}$ 's are isotopic to leaves of a given lamination $L \in \mathscr{A} \mathscr{L}(M)$, we may assume they have been isotoped to coincide with leaves. Apply Proposition 1.2 with $C$ the remaining $C_{i}$ 's. Then $L$ meets each $N\left(C_{i}\right)$ either in transverse arcs, parallel circles, or rays spiralling in on $C_{i}$, and in each $P_{j}$, if we pinch away the product complementary regions of $L$ in $P_{j}$ we obtain a lamination $L_{j} \in \mathscr{A} \mathscr{L}\left(P_{j}\right)$. (In terms of prelaminations, we may represent $L$ by a prelamination meeting each $P_{j}$ in a prelamination representing $L_{j}$.) In this situation we say $L$ is in normal form with respect to the given decomposition of $M$ into pairs of pants.

The universal abelian cover $\widehat{P}_{j}$ covers the lifts of $P_{j}$ to $\widehat{M}$, so 


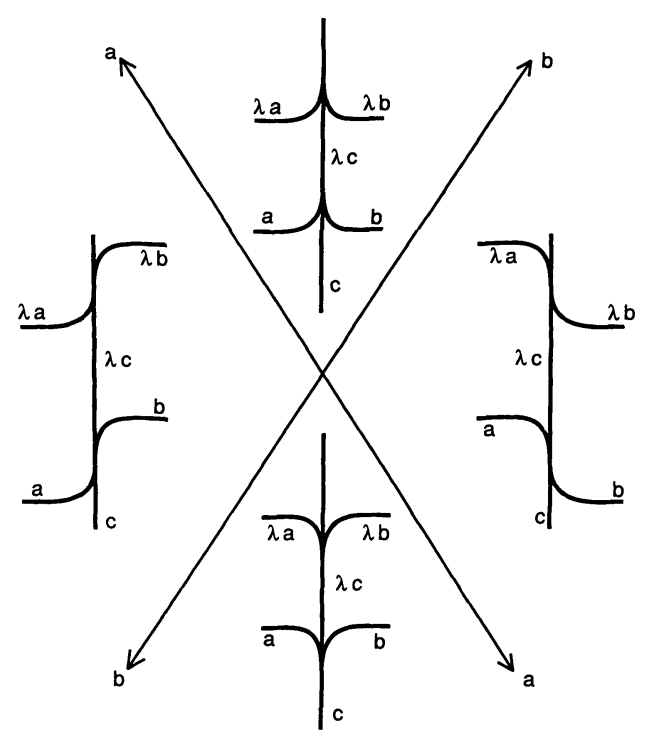

FIGURE 3.2

by the previous special case there are loops in $\widehat{P}_{j}$, hence also in the lifts of $P_{j}$ to $\widehat{M}$, whose length functions determined the restriction of $\widehat{L}$ to these lifts. In particular, holonomy is determined by length functions. It remains to consider how the restrictions of laminations to the $P_{j}$ 's extend across the $C_{i}$ 's.

Consider first a two-sided $C_{i}$ which does not bound in $M$, so the holonomy around $C_{i}$ may be non-trivial. Four "connector" tracks in a lift $\widehat{N}\left(C_{i}\right)$ to $\widehat{M}$ are shown in Figure 3.2.

The weights $a, b, c$, together with the holonomy $\lambda$ around $C_{i}$ determine all the other weights. In the upper connector the space of weights is the solution set $((a, b, c, \lambda) \mid a+b+c=\lambda c)$, while in the left connector it is the solution set for $a+c=b+\lambda c$; the other two connectors are symmetric. When some of the weights $a, b$, or $c$ are zero, the various connectors have certain common subtracks. This means the four solution sets fit together to form a single "connector space" $\mathscr{C}_{i}$. Projectivizing by factoring out by scalar multiplication of the weights $(a, b, c) \neq(0,0,0)$ for each fixed $\lambda$ yields a space $\mathscr{P} \mathscr{C}_{i}$. In the upper and lower connectors $c \neq 0$ (for non-empty laminations), so we may projectivize by setting $c=1$, yielding the equation $\lambda=a+b+1$ for the upper connector. This connector thus contributes a quadrant to $\mathscr{P} \mathscr{C}_{i}$, the upper quadrant of Figure 3.2 in fact, with $\lambda$ essentially the vertical coordinate. The lower connector is similar. For the left connector, if $c \neq 0$ we again projectivize 
by setting $c=1$, obtaining the relation $\lambda=a-b+1$ and the left quadrant with $\lambda$ again the vertical coordinate. And similarly for the right quadrant. For both these quadrants $c$ can be zero, in which case $a=b$ and $\lambda$ is arbitrary. This yields a common line at infinity, to the left of the left quadrant and to the right of the right quadrant. Thus $\mathscr{P} \mathscr{C}_{i}$ is just a cylinder $S^{1} \times \mathbb{R}_{+}$with $\lambda$ the second coordinate. "Unprojectivizing," each $S^{1} \times(\lambda)$ becomes an $\mathbb{R}^{2} \times(\lambda)$, so $\mathscr{C}_{i}$ is $\mathbb{R}^{2} \times \mathbb{R}_{+}$.

All ways of extending affine laminations over $N\left(C_{i}\right)$ (in normal form) are carried by the four connector tracks. Namely, the upper and lower connectors take care of spiralling into $C_{i}$. And for laminations crossing $C_{i}$ transversely, if $\lambda \neq 1$ there is a unique extension across $C_{i}$ since in the cover $\widehat{N}\left(C_{i}\right)$ the structure along $\widehat{C}_{i}$ is isometric to $(0, \infty)$, which has no isometries; since the left and right connectors carry some extension with given $a, b$, and $\lambda \neq 1$ ( $c$ being determined by the equation $c=|(a-b) /(\lambda-1)|)$, it must be the unique one. For $\lambda=1$ the extension is not unique since variable twisting along $C_{i}$ is possible, but the left and right connectors suffice here, just as in the measured lamination case.

Coming back to proving injectivity of length functions, consider first the case that $\lambda \neq 1$. For the upper and left connectors we can then solve their defining equations for $c=(a \pm b) /(\lambda-1)$, the plus sign for the upper connector and the minus sign for the left connector. Since length functions $l_{\gamma}$ for $\gamma$ 's in the $\widehat{P}_{j}$ 's determine the holonomy $\lambda$ and the weights $a$ and $b$, these $l_{\gamma}$ 's suffice to distinguish the laminations carried by any one of the four connectors (still assuming $\lambda \neq 1$ ). However, these $l_{\gamma}$ 's do not distinguish the two-fold ambiguity between laminations carried by the upper and left connectors with the same $a, b$, and $\lambda$-essentially, the choice of sign in the formula for $c$ above. (The ambiguity is no more than two-fold: The upper and lower connectors are distinguished by whether $\lambda>1$ or $\lambda<1$, and with fixed $\lambda \neq 1$ the left and right connectors are distinguished by whether $a>b$ or $a<b$; when $a=b$, then $c=0$ and we have laminations carried by a common subtrack.)

To resolve this two-fold ambiguity we use a certain loop $\gamma$ which crosses $\widehat{C}_{i}$, indicated in Figure 3.3 . Here the cover $\widehat{P}_{j}$ for the $P_{j}$ on one side of $C_{i}$ is shown. The $P_{k}$ on the other side of $C_{i}$ gives another cover $\widehat{P}_{k}$ glued to this one along the lift $\widehat{C}_{i}$ of $C_{i}$. The loop $\gamma$ consists of two symmetric arcs in $\widehat{P}_{j}$ and $\widehat{P}_{k}$, with the property that each arc meets all $\alpha$ and $\beta$ curves minimally. We pinch together the 


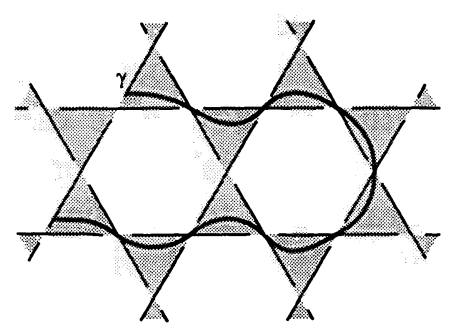

FIGURE 3.3

ends of the $\alpha$ and $\beta$ curves which meet $\widehat{C}_{i}$ between the two points of $\gamma \cap \widehat{C}_{i}$ to form the branch of the connector track with weight $a$ or $b$. The length of $\gamma$ is then $c+\lambda c$ (from its two intersections with $\widehat{C}_{i}$ ) plus the weights on certain $\alpha$ and $\beta$ curves in $\widehat{P}_{j}$ and $\widehat{P}_{k}$ disjoint from $\widehat{C}_{i}$. We know the latter weights and $\lambda$ are detectable by length functions, so $l_{\gamma}$ distinguishes between signs in the formula $c=(a \pm b) /(\lambda-1)$.

In case $\lambda=1$ we are essentially in the familiar situation of measured laminations near $C_{i}$, and the same curve $\gamma$ detects $c$, the amount of twisting around $C_{i}$. It does not determine the direction of twisting (the choice of left or right connector track), but as in [H], $\gamma$ together with its image under a Dehn twist around $C_{i}$ suffices for this.

For a two-sided $C_{i}$ which bounds in $M$ there is no holonomy, and the argument just given for the case $\lambda=1$ applies.

The situation for a one-sided $C_{i}$ is similar but somewhat simpler since the connector space $\mathscr{C}_{i}$ is $\mathbb{R} \times \mathbb{R}_{+}$instead of $\mathbb{R}^{2} \times \mathbb{R}_{+}$. There are the three connectors shown in Figure 3.4.

In the left connector $\lambda$ is arbitrary, and $a$ and $\lambda$ determine $c=$ $a /(1+\lambda)$. For the two connectors on the right, $\lambda \geq 1$ for the upper one and $\lambda \leq 1$ for the lower one, and $a$ and $\lambda$ determine $c=a / \pm(\lambda-1)$ unless $\lambda=1$, when $a=0$ and $c$ is arbitrary. Projectivizing, for fixed $\lambda$ there is a unique lamination carried by the left connector and
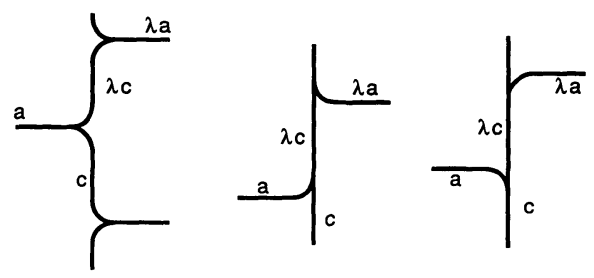

FIGURE 3.4 
a unique lamination carried by one of the right connectors, so $\mathscr{P} \mathscr{C}_{i}$ is $S^{0} \times \mathbb{R}_{+}$and hence $\mathscr{C}_{i}$ is $\mathbb{R} \times \mathbb{R}_{+}$. As before, all ways of extending laminations over $N\left(C_{i}\right)$ are carried by these three connectors. (When $\lambda=1$ laminations crossing $C_{i}$ transversely cannot twist non-trivially around $C_{i}$, just as in the measured lamination case.)

To show injectivity in the case of a one-sided $C_{i}$, when $\lambda \neq 1$ we just have to distinguish between the two laminations with the same $a$ and $\lambda$ which are carried by the left and right connectors in Figure 3.5. The same loop $\gamma$ used in the case of a two-sided $C_{i}$ suffices for this since it detects the weight $c\left(1+\lambda^{2}\right)$ and the laminations to be distinguished have different values of $c$. When $\lambda=1$ and $a=0$ this $\gamma$ also detects the weight $c$ on $C_{i}$.

4. Global structure of $\mathscr{\mathscr { L }}(M)$.

THEOREM 4.1. The map

$$
\sigma: \mathscr{A L}(M) \rightarrow \operatorname{Hom}\left(\pi_{1}(M), \mathbb{R}_{+}\right) \approx H^{1}(M ; \mathbb{R}), \quad L \mapsto \sigma_{L},
$$

is a trivial fiber bundle, with fiber $S^{-3 \chi-\beta-1} * \Delta^{\beta-1} \approx \mathscr{P} \mathscr{L}(M)$, where $\chi$ is the Euler characteristic and $\beta$ the number of boundary components of $M$.

Proof. Let $\widetilde{\mathscr{A} \mathscr{L}}(M)$ be the space of pairs $(\widehat{L}, \sigma) \in \widehat{\mathscr{A L}}(M) \times$ $H^{1}(M ; \mathbb{R})$ such that $\sigma=\sigma_{\widehat{L}}$ if $\widehat{L} \neq \varnothing$. This is a "fiberwise unprojectivization" of $\mathscr{A} \mathscr{L}(M)$ which artificially distinguishes different holonomies for the empty lamination. We shall show that the projection $\sigma: \widetilde{\mathscr{A} \mathscr{L}}(M) \rightarrow H^{1}(M ; \mathbb{R})$ is a product bundle with fiber $\mathbb{R}^{-3 \chi-\beta} \times[0, \infty)^{\beta} \approx \mathscr{M} \mathscr{L}(M)$.

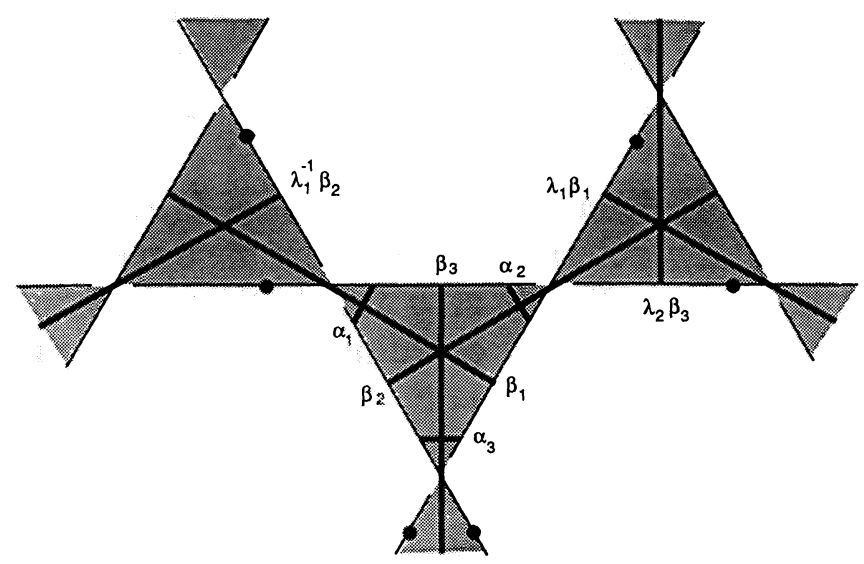

FIGURE 4.1 
We begin with the case that $M$ is a pair of pants. Figure 4.1 shows a part of the cover $\widehat{M}$ with chosen lifts of the arcs $\alpha_{i}$ and $\beta_{i}$, together with a few translates of these lifts, where $\lambda_{1}$ denotes translation by one unit to the right and $\lambda_{2}$ translation by one unit upward and to the right. Let $a_{i}$ and $b_{i}$ be the weights on $\alpha_{i}$ and $\beta_{i}$, and let $\lambda_{i}$ also denote the value of the holonomy $\sigma\left(\lambda_{i}\right)$. For the three arcs of $\partial \widehat{M}$ between the indicated pairs of dots the total weights are:

$$
\begin{aligned}
& w_{1}=a_{1}+a_{2}+\left(1+\lambda_{2}\right) b_{3}, \\
& w_{2}=a_{2}+a_{3}+\left(1+\lambda_{1}\right) b_{1}, \\
& w_{3}=a_{1}+a_{3}+\left(1+\lambda_{1}^{-1}\right) b_{2} .
\end{aligned}
$$

The weights $w_{i}$ and the $\lambda_{i}$ 's determine the weights $a_{i}$ and $b_{i}$. To see this there are four cases to consider: $b_{1}=b_{2}=b_{3}=0, a_{1}=b_{2}=$ $b_{3}=0, b_{1}=a_{2}=b_{3}=0$, and $b_{1}=b_{2}=a_{3}=0$. In the first case all three triangle inequalities hold among the $w_{i}$ 's, while in each of the other cases one triangle inequality is reversed. Solvability for $a_{i}$ 's and $b_{i}$ 's in terms of $w_{i}$ 's and $\lambda_{i}$ 's in all four cases is easily verified, for arbitrary $w_{i} \geq 0$ and $\lambda_{i}>0$. Thus we have a continuous bijection $[0, \infty)^{3} \times H^{1}\left(M ; \mathbb{R}_{+}\right) \rightarrow \widetilde{\mathscr{A} \mathscr{L}}(M)$ sending $\left(w_{1}, w_{2}, w_{3}, \lambda_{1}, \lambda_{2}\right)$ to the corresponding measured lamination in $\widehat{M}$. The inverse map is also continuous since the $a_{i}$ 's, $b_{i}$ 's, $\lambda_{i}$ 's, and hence also $w_{i}$ 's are expressible as algebraic combinations of length functions.

For the general case when $M$ is divided into pairs of pants $P_{j}$ by circles $C_{i}$, let $\mathscr{P}$ denote the subspace of the product $[0, \infty)^{\beta} \times$ $\Pi_{i} \mathscr{C}_{i} \times H^{1}(M ; \mathbb{R})$ consisting of points for which $\sigma\left(C_{i}\right)=\lambda_{i}$, where $\sigma \in H^{1}(M ; \mathbb{R})$ and $\lambda_{i}$ is the $\mathbb{R}_{+}$coordinate in $\mathscr{C}_{i} \approx \mathbb{R}^{2} \times \mathbb{R}_{+}$or $\mathbb{R} \times \mathbb{R}_{+}$. It follows that $\mathscr{P}$ is fiberwise (with respect to projection onto $\left.H^{1}(M ; \mathbb{R})\right)$ a product $[0, \infty)^{\beta} \times \mathbb{R}^{-3 \chi-\beta} \times H^{1}(M ; \mathbb{R})$ since there are $-3 \chi \quad P_{j}$ 's and each two-sided $C_{i}$ contributes an $\mathbb{R}^{2}$ to $\mathbb{R}^{-3 \chi-\beta}$, each one-sided $C_{i}$ an $\mathbb{R}$.

Each point $p \in \mathscr{P}$ determines a lamination $\widehat{L}(p)$ in $\widehat{\mathscr{A} \mathscr{L}}(M)$ as follows. In each $P_{j}, p$ determines weights on the three circles of $\partial P_{j}$, namely, the $[0, \infty)$ coordinates of $p$ for circles of $\partial P_{j}$ in $\partial M$, and for circles $\partial P_{j}$ in $N\left(C_{i}\right)$ the $\mathscr{C}_{i}$ coordinate of $p$ gives weights. As we have seen in the special case $M=P_{j}$ these weights on $\partial P_{j}$ together with the holonomy $\sigma$ determine a lamination in $P_{j}$. The $\mathscr{C}_{i}$ coordinates of $p$ also tell how to fit these laminations in the $P_{j}$ 's together to form a lamination $\widehat{L}(p)$ in $\widehat{M}$. By the results in the preceding section about length functions, the map $\mathscr{P} \rightarrow \widehat{\mathscr{A L}}(M), p \mapsto \widehat{L}(p)$, 
is a homeomorphism onto its image, which is all of $\widetilde{\mathscr{A} \mathscr{L}}(M)$ since laminations can be isotoped to be in normal form.

\section{REFERENCES}

[C] A. Casson, Automorphisms of surfaces after Nielsen and Thurston, notes by S. Bleiler, Univ. of Texas at Austin, 1983.

[FLP] A. Fathi, F. Laudenbach, and V. Poénaru, Travaux de Thurston sur les surfaces, Astérisque, 66-67 (1979).

[H] A. Hatcher, Measured lamination spaces for surfaces, from the topological viewpoint, Topology Appl., 30 (1988), 63-88.

[HP] J. Harer and R. Penner, Combinatorics of train tracks, preprint.

[T] W. Thurston, On the geometry and dynamics of diffeomorphisms of surfaces, Bull. Amer. Math. Soc., 19 (1988), 417-431.

Received November 15, 1989.

CORNELl UNIVERSITY

ITHACA, NY 14853

AND

RUTGERS UNIVERSITY

NEWARK, NJ 07102 



\section{PACIFIC JOURNAL OF MATHEMATICS EDITORS}

V. S. VARAdARAJAN

(Managing Editor)

University of California

Los Angeles, CA 90024-1555

Herbert Clemens

University of Utah

Salt Lake City, UT 84112

F. Michael CHRIST

University of California

Los Angeles, CA 90024-1555

ThOMAs ENRIGHT

University of California, San Diego

La Jolla, CA 92093
Nicholas ERCOLANI

University of Arizona

Tucson, AZ 85721

R. FINN

Stanford University

Stanford, CA 94305

VAUGHAN F. R. JoNES

University of California

Berkeley, CA 94720

SteVen KercKhofF

Stanford University

Stanford, CA 94305
C. C. MOORE

University of California

Berkeley, CA 94720

MARTIN SCHARLEMANN

University of California

Santa Barbara, CA 93106

HAROLD STARK

University of California, San Diego

La Jolla, CA 92093

\section{ASSOCIATE EDITORS}
R. Arens
E. F. BeCKenBACH
B. H. NeumanN
F. WolF
(1904-1989)
K. YoshidA (1906-1982)

TIONS

UNIVERSITY OF ARIZONA

UNIVERSITY OF BRITISH COLUMBIA

UNIVERSITY OF OREGON

UNIVERSITY OF SOUTHERN CALIFORNIA

CALIFORNIA INSTITUTE OF TECHNOLOGY

UNIVERSITY OF CALIFORNIA

MONTANA STATE UNIVERSITY

STANFORD UNIVERSITY

UNIVERSITY OF HAWAII

UNIVERSITY OF NEVADA, RENO

UNIVERSITY OF TOKYO

NEW MEXICO STATE UNIVERSITY

UNIVERSITY OF UTAH

OREGON STATE UNIVERSITY

WASHINGTON STATE UNIVERSITY

UNIVERSITY OF WASHINGTON 


\section{Pacific Journal of Mathematics}

Vol. 154, No. $1 \quad$ May, 1992

Richard Arens, Pseudo regular elements in a normed ring $\ldots \ldots \ldots \ldots \ldots 1$

Joan Birman and William W. Menasco, Studying links via closed braids.

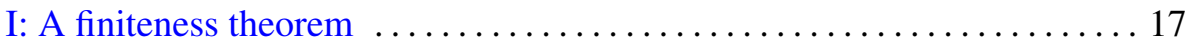

Etsurō Date, Michio Jimbo, Kei Miki and Tetsuji Miwa, Braid group

representations arising from the generalized chiral Potts models ....... 37

Toshihiro Hamachi, A measure theoretical proof of the Connes-Woods

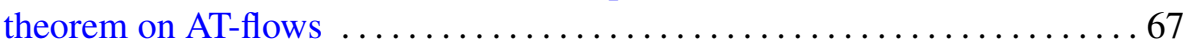

Allen E. Hatcher and Ulrich Oertel, Affine lamination spaces for surfaces ....................................... 87

David Joyner, Simple local trace formulas for unramified $p$-adic groups $\ldots .103$

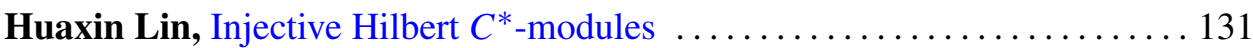

John Marafino, The boundary of a simply connected domain at an inner

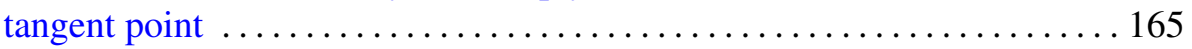

Gonzalo Riera and Rubi Rodriguez, The period matrix of Bring's curve . . 179 